\title{
Concordancia entre la Edad Cronológica y Edad Dental Según el Método de Demirjian en Pacientes Mexicanos
}

\author{
Concordance Between Chronological Age and Dental \\ Age by the Demirjian Method in Mexican Patients
}

\author{
Jorge Mario Ramirez Roman; Adriana Lucila Garza Ballesteros; Efigenia Moreno Terrazas; \\ Maria de Lourdes Verdugo Barraza; Jesus Hector Lopez Zamora \& Julio Carlos Garnica Palazuelos
}

RAMIREZ, R. J. M.; GARZA, B. A. L.; MORENO, T. E.; VERDUGO, B. M. L.; LOPEZ, Z. J. H. \& GARNICA, P. J. C. Concordancia entre la edad cronológica y edad dental según el método de demirjian en pacientes mexicanos. Int. J. Odontostomat., 12(4):412-415, 2018.

RESUMEN: En la práctica forense un dato de gran importancia es la edad cronológica de los pacientes, este puede obtenerse de múltiples formas, algunas son complejas, costosas y tardadas, el cálculo de la edad dental (ED) por vía radiográfica es sencillo y de bajo costo, ha mostrado ser concordante con la edad cronológica (EC), presentando variaciones por sexo y raza. El objetivo de este estudio es establecer la concordancia de la ED y la EC en población mexicana. Este es un estudio transversal, observacional en el que un residente estandarizado evaluó 88 Ortopantomografías (44 femeninos y 44 masculinos) de manera cegada respecto a la EC, del archivo de la clínica de Ortodoncia de la Facultad de Odontología de la Universidad Autónoma de Sinaloa, con edades desde los 3 a los 16 años. Existe una diferencia en meses de 3,36 entre los valores ED y EC en el total de la población $(p=0,384)$ con una concordancia de $94 \%$ entre los valores. Al analizarlos por sexo los masculinos muestran una diferencia de 5,16 ( $P=0,785)$ con una concordancia de $96 \%$ y los femeninos de $7,32(P=0,095)$ con una concordancia de $92 \%$. La ED según Demirjian es un método confiable para estimar la EC en esta población.

PALABRAS CLAVE: edad dental, confiabilidad, método de demirjian, mexicanos. radiográfico.

\section{INTRODUCCIÓN}

Conocer la edad cronológica aproximada de pacientes con fecha de nacimiento desconocida es una problemática forense que ayuda a la identificación de pacientes pediátricos que se encuentran en situación de calle o han sido desprendidos de sus padres por algún motivo, así como en aquellos que han perdido la vida y requieren de ser identificados, una prueba que nos estime aproximadamente este dato es de gran utilidad para disminuir la cantidad de posibles sujetos al momento de analizar los datos de este tipo de pacientes. La edad cronológica no es lo mismo que la edad biológica, ya que estas diferirán en más de $+/-1$ años el $32 \%$ y en más de $+/-2$ años aproximadamente el $5 \%$ de las veces. La maduración esquelética, sexual y somática están interrelacionadas y controla- das por el mismo factor o factores, la maduración dental no se correlaciona con las anteriores ya que dependerá de la calcificación de los dientes (Buschang et al., 2017).

La maduración dental (MD) es un proceso de calcificación de la dentición temporal y permanente de un individuo, que comprende la formación de las primeras cúspides y bordes incisales de la dentición temporal hasta el cierre apical de los últimos molares en la dentición permanente (Demirjian et al., 1973; Catala \& García, 2004), no se altera por factores locales y ambientales (Fanning, 1961) (Sapoka \& Demirjian, 1971), aunque tiene momentos de inicio distintos por sexo (Águila, 1991), se ha reportado lige- 
ras alteraciones en la MD de acuerdo a la raza u origen étnico (Águila) sin embargo una vez iniciada no se detiene lo que permite su asociación con la edad cronológica en niños (Águila).

Se han descrito diversos métodos para el estudio de la maduración dental, clasificándose de diferentes formas, como la madurez de la dentición (Feijóo García, 2010; Paz Cortés, 2013), métodos de radiografías múltiples como el de Moorees (Moorrees et al., 1963) o Nolla (1960) que establecen estadios de maduración para cada diente evaluando la magnitud de calcificación dentaria, métodos que evalúan la calcificación y la morfología de la cámara pulpar (Kvaal \& Solheim, 1994) y de la radiografía panorámica como el descrito por Demirjian et al. y Demirjian \& Goldstein (1976), que evalua la magnitud de calcificación, morfología de la cámara pulpar y desarrollo radicular de los dientes del cuadrante inferior izquierdo excepto el tercer molar en la Ortopantomografía, se describen 8 estadios madurativos para cada diente, ordenados alfabéticamente de la $\mathrm{A}$ hasta la $\mathrm{H}$ mediante criterios visuales y escritos, predominando en la valoración los criterios escritos sobre los visuales.

El objetivo de este estudio fue analizar la concordancia entre la edad cronológica y la edad dental calculada con el método de Demirjian.

\section{MATERIAL Y MÉTODO}

Se seleccionaron 88 pacientes del archivo de la clínica de la Maestría de Ortodoncia y ortopedia de la Facultad de odontología de la Universidad Autónoma de Sinaloa, con edades desde los 3 a los 16 años que contaran con radiografía panorámica. Se incluyeron paciente sin síndromes genéticos diagnosticados en su expediente, ni alteraciones metabólicas o crónico-degenerativas en su expediente. Se excluyeron radiografías panorámicas en mal estado, agenesia dental de algún órgano dental excepto el tercer molar, con tratamientos de endodoncia, todos en el cuadrante a evaluar y aquellos con tratamiento ortopédico $u$ ortodóncico previo.

Realización de la prueba: se estandarizo a un residente de ortodoncia para identificar de manera repetida los estadios dentales según el método de Demirjian con un coeficiente de correlación intraclase $(\mathrm{CCl})$ de $(0,9)$, se revisó la calidad de apreciación de las estructuras a evaluar, la edad cronológica se obtuvo del expediente y fue resguardada; la muestra calculada fue de 88 sujetos, para encontrar diferencias entre 2 medias relacionadas, esperando encontrar una diferencia de $0,3( \pm 1,0)$ años de edad dental respecto a la edad cronológica con un a de 0,05 y un b de 0,8. La evaluación de la edad dental se calculó de acuerdo

A) Calcificación inicial de la corona
B) Superficie oclusal Fusionada
C) Superficie oclusal formada
D) Formación de la corona completa hasta
la unión cemento-esmalte
E) Diente unirradicular:longitud radicular menor
que el tamaño de la corona (en molares la
bifurcación ya es visible)
F) La longitud radicular es igual al largo de la
corona
G) El ápice de los canales radiculares está
parcialmente abierto

El ápice de los canales radiculares está cerrado

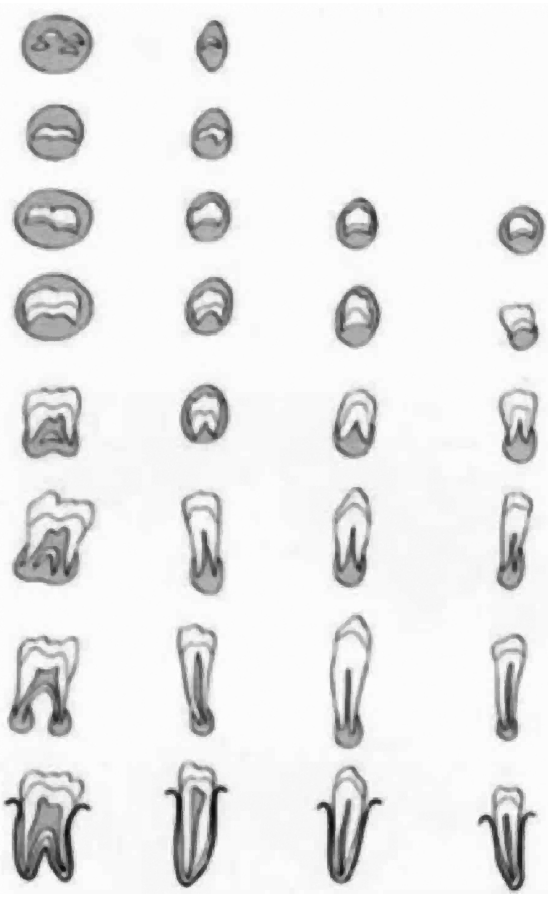

Fig. 1. Estadios de madurez dental de acuerdo al método de Demirjian. 
RAMIREZ, R. J. M.; GARZA, B. A. L.; MORENO, T. E.; VERDUGO, B. M. L.; LOPEZ, Z. J. H. \& GARNICA, P. J. C. Concordancia entre la edad cronológica y edad dental según el método de demirjian en pacientes mexicanos. Int. J. Odontostomat., 12(4):412-415, 2018.

al Índice de Demirjian (Fig. 1) (Demirjian et al.), de manera cegada sobre la edad cronológica, un PHD fungió como evaluador independiente procesando los datos edad dental y cronológica, se calculó las diferencias y concordancias entre las dos mediciones a través de coeficientes de correlación intra clase.

\section{RESULTADOS}

Se analizaron: 88 ortopantomografías, 44 de sexo femenino y 44 sexo masculino. Con edades cronológicas de 3,7 a 16,0 años, y 3,4 a 16,0 de edad dental. No hubo diferencias en el promedio de edad cronológica por sexos masculinos 11,0991 (+/-3,2), Femeninos $10,9273(+/-2,68)(p=0,787)$ y en edad dental masculinos $11,5295(+/-3,07)$, Femeninos $11,5295(+/-2,65)(p=0,440)$, al evaluar diferencias entre edad cronológica y dental no hubo diferencias en masculinos edad cronológica 11,0991 (+/-3,2), edad dental $11,5295(+/-3,07)(p=0,785)$, ni en femeninos edad cronológica $10,9273(+/-2,68)$, edad dental $11,5295( \pm 2,65)(p=0,095)$. ni en toda la población edad cronológica 11,0132 (+/-2,95), edad dental 11,2920 (+/ $-2,86)(p=0,384)$. Los $\mathrm{CCl}$ entre edad cronológica y dental fueron de masculinos 0,962 , femeninos 0,923 y en total 0,944 (Tabla I).

\section{DISCUSIÓN}

La maduración esquelética, sexual y somática están interrelacionadas y los cambios hormonales, nutricionales, de crecimiento etc. Lo alteran lo que genera gran variabilidad y dificulta su uso como indicador de edad cronológica. No así la maduración dental, que dependerá de la magnitud de calcificación de los dientes, este proceso ocurre de manera constante sin alterarse por factores hormonales o nutricionales (Buschang et al.). Se sabe que el método de Demirjian es un excelente indicador de edad cronológica se ha probado en poblaciones Iranís (Bagherian \& Sadeghi, 2011), Saudíes (Al-Dharrab et al., 2017), Españoles (Delgado Tomás, 2014), Indues (Malik et al., 2012) y tailandeses (Duangto et al., 2016) encontrando que es una herramienta útil; sin embargo estas aseveraciones son inexactas ya que se calcularon mediante correlaciones, las cuales estadísticamente no son el método de elección para probar concordancia o validez (Cortés-Reyes et al., 2010), existen reportes de que la edad dental es variable por razas incluso se ha ajustado el método de Demirjian a otras poblaciones, sin embargo el procedimiento estadístico no es concreto (Willems et al., 2001). Los hallazgos en población mexicana son bastante concordantes ( $\mathrm{CCl}$ masculinos 0,962 , femeninos 0,923 y ambos sexos 0,944 ) por lo que se puede pensar que la herramienta es útil en esta población, sin embargo el rango de edad fue limitado por lo que una población más amplia seria de utilidad.

La edad dental de acuerdo al método de Demirjian mostró ser un buen indicador de la edad cronológica, con una excelente concordancia en esta población, por lo que pudiera considerarse una prueba tamiz en los análisis forenses de pacientes con fecha de nacimiento desconocida.

AGRADECIMIENTOS: Maestría en Ortodoncia y Ortopedia de la Facultad de Odontología, Universidad Autónoma de Sinaloa. Programa Nacional de Posgrados de Calidad CONACyT.

RAMIREZ, R. J. M.; GARZA, B. A. L.; MORENO, T. E.; VERDUGO, B. M. L.; LOPEZ, Z. J. H.; GARNICA, P. J. C. Concordance between chronological age and dental age by the Demirjian method in Mexican patients. Int. J. Odontostomato., 12(4):412-415, 2018.

ABSTRACT: In forensic practice knowledge of chronological age (CA) is an important factor, and it can be estimated by several methods. Some are complex, expensive and time consuming. Dental Age (DA) estimation by radiographic methods is easy and inexpensive, it has shown to be consistent with chronological age (CA), with variations according to sex and race. The objective of this study was to estimate the concordance between DA and CA in Mexicans. This is a cross sectional, observational study, in which a standardized dental resident reviewed 88

Tabla I. Estadística descriptica expresada en años y coeficientes de correlación intraclase.

\begin{tabular}{lccccc}
\hline Genero & $\mathrm{N}$ & Edad Cronológica (D,S, ) & Edad Dental (D,S) & $\mathrm{X}^{2}$ & $\mathrm{CCl}$ \\
\hline Masculino & 44 & $11,0991(+/-3,2)$ & $11,5295(+/-3,07)$ &, 785 & 0,962 \\
Femenino & 44 & $10,9273(+/-2,68)$ & $11,5295(+/-2,65)$ &, 095 & 0,923 \\
Total & 88 & $11,0132(+/-2,95)$ & $11,2920(+/-2,86)$ &, 384 & 0,944 \\
\hline
\end{tabular}


Orthopantomographies (44 female, 44 male), blinded to CA, of the Orthodontics School Files of the Universidad Autónoma de Sinaloa, the population was 3 to 16 years of age. The main difference in months between DA and CA was 3.36 $(p=0.384)$ and $94 \%$ of concordance, the analysis by sex for males showed a main difference of $5.16(P=0.785)$ and 96 $\%$ concordance; for females it showed $7.32(\mathrm{P}=0.095)$ and concordance of $92 \%$. In conclusion the DA according to Demirjian is a reliable method to estimate CA in these patients.

KEY WORDS: dental age, reliability, Demirjian method, Mexicans, radiographic.

\section{REFERENCIAS BIBLIOGRÁFICAS}

Águila, F. J. Crecimiento Craneofacial: Ortodoncia y Ortopedia. Barcelona, Aguiram, 1991.

Al-Dharrab, A. A.; Al-Sulaimani, F. F.; Bamashmous, M. S.; Baeshen, H. A. \& Zawawi, K. H. Radiographic evaluation of dental age maturity in 3-17-years-old saudi children as an indicator of chronological age. J. Orthod. Sci., 6(2):47-53, 2017.

Bagherian, A. \& Sadeghi, M. Assessment of dental maturity of children aged 3.5 to 13.5 years using the Demirjian method in an Iranian population. J. Oral Sci., 53(1):37-42, 2011.

Buschang, P. H.; Roldan, S. I. \& Tadlock, L. P. Guidelines for assessing the growth and development of orthodontic patients. Semin. Orthod., 23(4):321-5, 2017.

Catala, A. \& García, C. Crecimiento Craneofacial y Desarrollo de Las Arcadas Dentarias. Barcelona, Masson, 2004. pp.37-54.

Cortés-Reyes, E.; Rubio-Romero, J. A. \& Gaitán-Duarte, H. Métodos estadísticos de evaluación de la concordancia y la reproducibilidad de pruebas diagnósticas. Rev. Colomb. Obstet. Ginecol., 61(3):247-55, 2010.

Delgado Tomás, L. F. Estimacion de la Edad Cronologica a través de los Métodos de Demirjian y Nolla en una Muestra Portuguesa y Española. Tesis. Santiago de Compostela, Universidad de Santiago de Compostela, 2014.

Demirjian, A. \& Goldstein, H. New systems for dental maturity based on seven and four teeth. Ann. Hum. Biol., 3(5):411-21, 1976.

Demirjian, A.; Goldstein, H. \& Tanner, J. M. A new system of dental age assessment. Hum. Biol., 45(2):211-27, 1973.

Duangto, P.; Janhom, A.; Prasitwattanaseree, S.; Mahakkanukrauh, P. \& lamaroon, A. New prediction models for dental age estimation in Thai children and adolescents. Forensic Sci. Int., 266:583.e1-583.e5, 2016.

Fanning, E. A longitudinal study of tooth formation and root resorption. N. Z. Dent. J., 57:202-17, 1961.

Feijóo García, G. Cronología de La Odontogénesis de Los Dientes Permanentes En Niños de La Comunidad de Madrid. Aplicación a La Estimación de La Edad Dentaria. Tesis Doctoral. Madrid, Universidad Complutense de Madrid, Facultad de Odontología, 2010.

Kvaal, S. \& Solheim, T. A non-destructive dental method for age estimation. J. Forensic Odontostomatol., 12(1):6-11, 1994.

Malik, P.; Saha, R. \& Agarwal, A. Applicability of Demirjian's method of age assessment in a North Indian female population. Eur. J. Paediatr. Dent., 13(2):133-5, 2012.

Moorrees, C. F.; Fanning, E. A. \& Hunt, E. E. Jr. Age variation of formation stages for ten permanent teeth. J. Dent. Res., 42:1490$502,1963$.
Nolla, C. M. The development of the permanent teeth. J. Dent. Child., 27:254-66, 1960.

Paz Cortés, M. M. Maduración y Desarrollo de Los Dientes Permanentes en Niños de la Comunidad de Madrid: Aplicación a la Estimación de la Edad Dentaria. Tesis Doctoral. Madrid, Universidad Complutense de Madrid, Facultad de Odontología, 2013.

Sapoka, A. A. \& Demirjian, A. Dental development of the French Canadian child. J. Can. Dent. Assoc. (Tor.), 37(3):100-4, 1971.

Willems, G.; Van Olmen, A.; Spiessens, B. \& Carels, C. Dental age estimation in Belgian children: Demirjian's technique revisited. J. Forensic Sci., 46(4):893-5, 2001.

Dirección para correspondencia:

Julio Carlos Garnica Palazuelos

Alvaro Obregon \#1880-43k

Culiacan Sinaloa

Colinas de San Miguel

Sinaloa

MÉXICO

Email: Julioka_garnica@hotmail.com

Recibido : 22-06-2018

Aceptado: 31-08-2018 